\title{
NONLOCAL ANALYSIS OF SINGLE AND DOUBLE-LAYERED GRAPHENE CYLINDRICAL PANELS AND NANO-TUBES UNDER INTERNAL AND EXTERNAL PRESSURES CONSIDERING THERMAL EFFECTS
}

\author{
SHAHRIAR DASTJERDI \\ Young Researchers and Elite Club, Mashhad Branch, Islamic Azad University, Mashhad, Iran \\ e-mail: dastjerdi_shahriar@yahoo.com \\ Massoumeh Lotfi, Mehrdad Jabbarzadeh \\ Department of Mechanical Engineering, Mashhad branch, Islamic Azad University, Mashhad, Iran
}

\begin{abstract}
Mechanical behavior of a bilayer graphene cylindrical panel and nano-tube is studied based on nonlocal continuum mechanics with regard to this aim, von-Karman assumptions and nonlocal theory of Eringen are considered. Then, the governing equations and boundary conditions have been derived applying energy method. While analyzing the bilayer cylindrical panel, the van der Waals interaction between the layers is considered in calculations. The constitutive equations are developed for nano-tubes under internal and external pressures. In order to solve the governing equations, the semi-analytical polynomial method (SAPM), which was presented by the authors before, is utilized and bending behavior of bilayer cylindrical panels and nano-tubes is investigated. Finally, the effects of temperature, boundary conditions, elastic foundation, loading, van der Waals interaction between the layers and single layer to bilayer analyses are studied for graphene cylindrical panels and nano-tubes.
\end{abstract}

Keywords: cylindrical graphene panels, nano-tube, nonlocal elasticity theory, first-order shear deformation theory

\section{Introduction}

Shells and panels are the most frequently applied engineering structures and are categorized in several groups. The curvature of shells is one basis of the categorization which is most significant in geometric shapes (cylindrical, conical, and spherical, etc.) and related equations. Another basis of the categorization is thickness of the shell, which divides into two groups of low thickness (shell) and high thickness (plate), for the thickness-to-radius ratio of $(h / R)_{\max } \leqslant 1 / 20$, it is assumed to be a low thickness shell. According to ever-increasing application of shells in industries, the significance of studying behavior of shell structures in different cases of loading including bending and buckling becomes more pronounced. One interesting nano structure which scientifics are devoted to is the carbon nano-tube. The tubes were discovered in 1991 (Iijima, 1991). Single wall carbon nano-tubes can be assumed as 2D single layer graphene sheets which are rolled. The extraordinary attributes of graphene sheets, carbon nano-tubes and their exclusive specifications made them a reason to progress and renovation of difference researches and nano-science (Tans et al., 1997; Martel et al., 1998; Postma et al., 2001). Experimental tests in nano scale are difficult and expensive. Therefore, developing a suitable mathematical model for nano structures is important. Continuum mechanics model is an appropriate and a low-cost solution. Disadvantages of the continuum method is that it is not possible to estimate the exact small scale effects. When the size of structures is small, the effects of small scale on mechanical behavior are very important. To eliminate the disadvantage in the classic continuum model, Eringen and Edelen (1972) presented a nonlocal elasticity theory and modified the classical continuum mechanics to consider the effects of small size. By considering the effects of size in 
related equations as material properties, the nonlocal theory of Eringen is extremely effective in presenting an exact and appropriate model of nano structures. Hence to study behavior of nano plates in many presented studies, the nonlocal theory is applied.

Murmu and Pradhan (2009) applied the theory of nonlocal elasticity to rectangular single layer graphene sheets in elastic environment. They applied both models of Winkler and Pasternak to simulate reactions of graphene sheets with their elastic environment. Reddy and Pang (2008) studied bending and buckling of carbon nano-tubes based on nonlocal theory. Aghababaei and Reddy (2009) investigated bending and free vibration equations based on the third-order shear deformation theory/ The nonlocal effects were then solved analytically for a isotropic rectangular sheet with simple boundary conditions. They concluded that two first order and third order shear deformation theories had similar results while related results from nonlocal theory of thin shell were difference. Pradhan (2009) analyzed buckling of isotropic graphene rectangular plates based on the third-order shear deformation theory and the Navier method. He concluded that by increasing the small scale effects or decreasing length of the plate, the ratio of nonlocal buckling loads to the local case would be increased. The first solution of the buckling problem of cylindrical shells under uniform lateral pressure was presented by Brush and Almroth (1975). Then many extensive researches on buckling of cylindrical shells by different materials and different boundary conditions have been done. Hoff and Soong (1965) studied buckling of a cylindrical shell under axial load in different conditions. They showed that if the ratio of shell length to its thickness was large enough, the value of stress in buckling mode would always be independent from length of the shell and the maximum critical stress would occurr for simply supported boundary conditions. Linghai et al. (2008) considered buckling of a cylindrical panel under axial load by a differential quadrature method in which the load was perpendicular to the surface. Zhao and Liew (2009) analyzed a cylindrical panel made of a functionally graded material (FGM) under thermal and dynamic loads based on the first order nonlinear shear deformation theory by applying the free element method. Nguyen and Hoang (2010) studied stability of a cylindrical panel made of a functionally graded material under axial pressure and simple supported boundary conditions. It was found that by increasing the ratio of length to radius of the cylindrical panel, critical load always increased. Khazaeinejad et al. (2010) analyzed buckling of cylindrical shells made of a functionally graded material under both axial loads and internal pressure by using the first order shear deformation theory. They concluded that by decreasing the power factor of material properties, critical load increased.

Lancaster et al. (2004) investigated the effect of imperfections on the buckling load in the form of local initial stress, which were probably more typical in practice than in purely geometric ones. Bisagni and Cordisco (2003) experimentally studied buckling and post-buckling behavior of four unstiffened thin-walled CFRP cylindrical shells for which the test equipment allowed application of axial and torsional loadings. Their results identified the effect of laminate orientation, showed that the buckling loads were essentially independent of the load sequence and demonstrated that the shells were able to sustain load in the post-buckling field without any damage. Degenhardt et al. (2010) performed buckling tests and buckling simulations on CFRP cylindrical shells to investigate the imperfection sensitivity and to validate the applied simulation methodologies. Fazelzadeh and Ghavanloo (2014) purused vibration characteristics of curved graphene ribbons (CGRs) embedded in an orthotropic elastic shell and investigated that there was significant dependence of natural frequencies on the curvature change. Biswas (2014) presented nonlinear analysis of plate and shell structures under mechanical and thermal loadings.

Ansari et al. (2016) studied size-dependent nonlinear mechanical behavior of third-order shear deformable functionally graded microbeams using the variational differential quadrature method. They used the gradient elasticity theory in their study. Zhang et al. (2016) applied FSDT element free IMLS-Ritz method to analyze free vibration of triangular CNT-reinforced composite plates subjected to in-plane stresses. 
Dastjerdi et al. (2016a) derived constitutive equations for graphene plates in Cartesian and cylindrical coordinate systems based on the nonlocal first and higher order shear deformation theories. Zhang et al. (2015) presented a nonlocal continuum model for vibration of single-layered graphene sheets based on the element-free kp-Ritz method. All the mentioned solving methods are popular in analyzing the small scale effects of nano structures. Continuing, Dastjerdi et al. (2016b,c) worked on static analysis of single layer annular/circular graphene sheets (Dastjerdi et al., 2016b) and the effect of vacant defects on bending analysis of circular graphene sheets (Dastjerdi et al., 2016c) based on the nonlocal theory of Eringen. They presented a new semianalytical polynomial method (SAPM) for solving ordinary and partial differential equations.

In this paper, for the first time, the nonlinear bending analysis of bilayer graphene cylindrical panels and nano-tubes has been studied. Nonlocal constitutive equations have been derived and solved applying SAPM (Dastjerdi et al., 2016c). It has been tried to consider all effective parameters on study such as boundary conditions, nonlocal parameter, temperature, loading, elastic foundation, etc.

\section{Nonlocal theory}

Different non-classical theories have been presented to analyze structures in nano and micro scales such as strain-gradient theories, couples stress and the nonlocal theory of Eringen. With regard to considering the simplicity of the equations and satisfactory results of the nonlocal theory, this theory has been applied by many researchers to analysis of mechanical behavior of nano structures. In other words, this theory has been applied in the present article, considering its advantages. According to the nonlocal theory of Eringen, stress in a specified point of surface is a function of strain in all points of the surface. As below in the integral equation, it is shown as a function of local stress (Eringen and Edelen, 1972)

$$
\sigma_{i j}=\int_{V} \alpha\left(\left|\chi^{\prime}-\chi\right|\right) \sigma_{i j}^{L}\left(\chi^{\prime}\right) d V\left(\chi^{\prime}\right)
$$

The integral must be calculated on the total surface. $\chi$ is a point whose stress can be calculated according to stress of all $\chi^{\prime}$ points of the structure. The indices $i, j$ are the same for $x, y, z$ of the shell coordinate components. The distance between $\chi$ and $\chi^{\prime}$ can be presented by $\left|\chi^{\prime}-\chi\right|$ and $\alpha\left(\left|\chi^{\prime}-\chi\right|\right)$, which are nonlocal weight functions. $\sigma_{i j}^{L}$ and $\sigma_{i j}^{N L}$ are local and nonlocal stresses, respectively. Finally, Eq. (2.1) can be expressed for the 2D case as

$$
\left(1-\mu \nabla^{2}\right) \sigma_{i j}^{N L}=\sigma_{i j}^{L} \quad \mu=\left(e_{0} a\right)^{2} \quad \nabla^{2}=\frac{\partial^{2}}{\partial x^{2}}+\frac{\partial^{2}}{\partial y^{2}}
$$

In the above, $a$ is related to internal dimensions and small size, $e_{0}$ is the material coefficient which results from experimental studies. The value of the nonlocal parameter $\mu$ is not specified exactly and is dependent on boundary conditions, mode number, layer quantity and type of movement (Eringen and Edelen, 1972). Wang and Wang (2007) illustrated that the value of the nonlocal parameter $\left(e_{0} a\right)$ is between 0 and $2 \mathrm{~nm}$. Consequently, many researchers have considered this range of the nonlocal parameter in their investigations. As a result, in this paper, the nonlocal parameter is assumed between 0 and $2 \mathrm{~nm}$, too.

\section{Governing equations}

A bilayer graphene cylindrical panel is considered as shown in Fig. 1, resting on a Winkler-Pasternak elastic medium under uniform transverse loading $q$. The Winkler-Pasternak stiffness coefficients are $k_{w}$ and $k_{p}$, respectively. Winkler considers the elastic foundation to be completely 


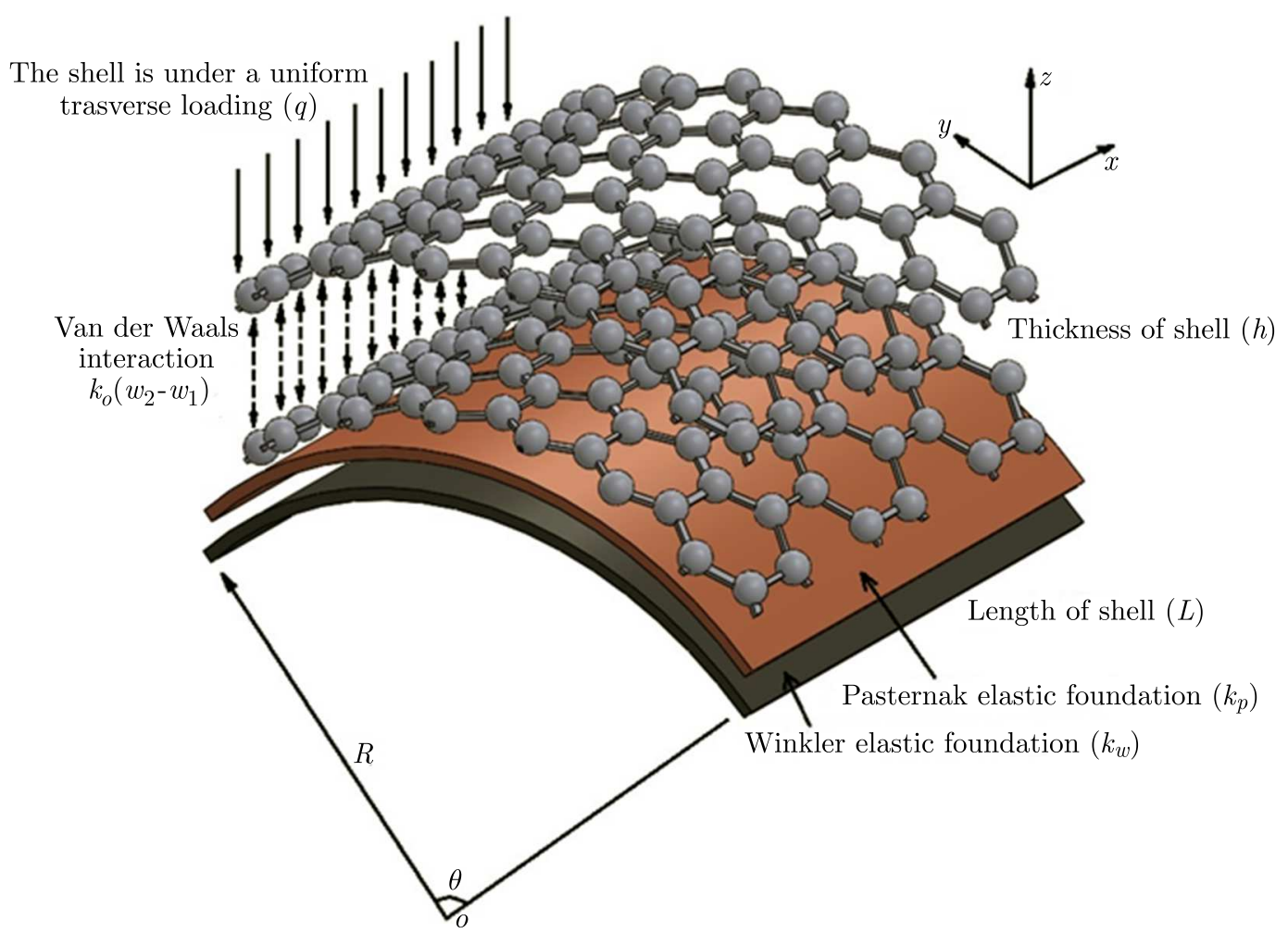

Fig. 1. Schematic view of a bilayer graphene cylindrical panel rested on Winkler-Pasternak elastic foundation

made of linear springs $\left(P=k_{w} w\right)$. If the mentioned foundation is affected by a transverse loading $q$, the springs would not be influenced beyond the loaded domain.

In the Pasternak elastic matrix, the effect of shear force among the spring elements is considered, which is completed by connecting the ends of the springs to the plate that only undergoes transverse shear deformation. The relationship between the load and deflection is obtained by assuming the vertical equilibrium of a shear layer $\left(P=k_{w} w+k_{p} \nabla^{2} w\right)$. The amount of van der Waals interaction depends on the distance between the layers. $k_{o}$ and $k_{o}^{*}$ can be introduced as a stiffness of spring force (van der Waals interaction) as $k_{o}(w 2-w 1)+k_{o}^{*}(w 2-w 1)^{3}$ in which $w 1$ represents defection of the upper layer and $w 2$ - the bottom layer. In practical investigations, the values of $w 1$ and $w 2$ are close to each other. So, the term $(w 2-w 1)^{3}$ is extremely insignificant. As a result, the non-linear term can be neglected for simplicity of calculations, and the van der Waals interaction can be considered linear as $k_{o}(w 2-w 1)$.

In this study, the constitutive equations are derived based on the first-order shear deformation theory (FSDT) in which the neglected assumptions in the classical plate theory are considered with more accuracy. According to the first-order shear deformation theory, the displacement field can be written as follows

$$
U i=u i+z \varphi i \quad V i=v i+z \psi i \quad W i=w i
$$

where $u i, v i$ and $w i$ are the displacement components of the mid-plane along the $x, y$ and $z$ directions, respectively. $\varphi i$ and $\psi i$ refer to the rotation functions of the transverse normal to $y$ and $x$ directions. The index $i=1,2$ refers to the upper and bottom layers, respectively. The strain filed is then as below 


$$
\begin{aligned}
& \varepsilon i_{x}=\frac{\partial u i}{\partial x}+\frac{1}{2}\left(\frac{\partial w i}{\partial x}\right)^{2}+z \frac{\partial \varphi i}{\partial x}-\alpha \Delta T \\
& \varepsilon i_{y}=\frac{w i}{R}+\frac{\partial v i}{\partial y}+\frac{1}{2}\left(\frac{\partial w i}{\partial y}\right)^{2}+z \frac{\partial \psi i}{\partial y}-\alpha \Delta T \\
& \gamma i_{x y}=\frac{\partial v i}{\partial x}+\frac{\partial u i}{\partial y}+\left(\frac{\partial w i}{\partial x} \frac{\partial w i}{\partial y}\right)+z\left(\frac{\partial \varphi i}{\partial y}+\frac{\partial \psi i}{\partial x}\right) \\
& \gamma i_{x z}=\varphi i+\frac{\partial w i}{\partial x} \quad \gamma i_{y z}=\psi i+\frac{\partial w i}{\partial y}-\frac{v i}{R}
\end{aligned}
$$

Now to obtain equilibrium equations, an energy method is applied in which variation of potential energy for external loads and strain energy must be zero

$$
\delta \pi=\delta U+\delta V=0 \quad \delta V=\iint_{A}\left(q-k_{w} w_{0}\right) \delta w_{0} d A
$$

and

$$
\delta U=\iiint_{A}\left(\sigma_{i j} \delta \varepsilon_{i j}\right) d v
$$

Equation (3.4) describes the strain energy which can be expanded as follows

$$
\delta U=\iiint_{A}\left(\sigma i_{x} \delta \varepsilon i_{x}+\sigma i_{y} \delta \varepsilon i_{y}+\sigma i_{x y} \delta \varepsilon i_{x y}+\sigma i_{y z} \delta \gamma i_{y z}+\sigma i_{x z} \delta \gamma i_{x z}\right) d v \quad i=1,2
$$

The stress and moment resultants are specified as follows

$$
\begin{aligned}
& {\left[N i_{x}, N i_{y}, N i_{x y}\right]^{N L}=\int_{-h / 2}^{h / 2}\left[\sigma i_{x}, \sigma i_{y}, \sigma i_{x y}\right]^{N L} d z} \\
& {\left[M i_{x}, M i_{y}, M i_{x y}\right]^{N L}=\int_{-h / 2}^{h / 2}\left[\sigma i_{x}, \sigma i_{y}, \sigma i_{x y}\right]^{N L} z d z} \\
& {\left[Q i_{y}, Q i_{x}\right]^{N L}=k_{s} \int_{-h / 2}^{h / 2}\left[\sigma i_{y z}, \sigma i_{x z}\right]^{N L} d z}
\end{aligned}
$$

where $k_{s}$ is the shear factor correction coefficient for the first-order shear deformation theory, which is taken 5/6. Now by substituting Eqs. (3.6) into Eq. (3.3) and integrating with respect to $z$, Eq. (3.3) can be developed. The obtained total energy equation must be equal to zero. The equilibrium equations are based on the nonlocal theory of Eringen. Applying Eq. (2.2) into the resultants (Eqs. (3.3)) and then substituting into the nonlocal governing equations, the constitutive equations can be obtained in the local form of the resultants considering the effects of the nonlocal parameter. Dastjerdi et al. (2016a) explained the process of obtaining the local form of governing equations from nonlocal ones. Finally, the nonlocal constitutive equations for the bilayer graphene cylindrical panel can be expressed as follows (some inconsiderable terms are neglected)

$$
\begin{array}{ll}
\frac{\partial N i_{x}^{L}}{\partial x}+\frac{\partial N i_{x y}^{L}}{\partial y}=0 & \frac{\partial N i_{y}^{L}}{\partial y}+\frac{Q i_{y}^{L}}{R}+\frac{\partial N i_{x y}^{L}}{\partial x}=0 \\
\frac{\partial M i_{x}^{L}}{\partial x}+\frac{\partial M i_{x y}^{L}}{\partial y}-Q i_{x}^{L}=0 & \frac{\partial M i_{y}^{L}}{\partial y}+\frac{\partial M i_{x y}^{L}}{\partial x}-Q i_{y}^{L}=0
\end{array}
$$


- upper layer

$$
\begin{aligned}
& -\frac{N 1_{y}^{L}}{R}+\frac{\partial Q 1_{y}^{L}}{\partial y}+\frac{\partial Q 1_{x}^{L}}{\partial x}+\left(1-\mu \nabla^{2}\right)\left[N 1_{x}^{L} \frac{\partial^{2} w 1}{\partial x^{2}}+2 N 1_{x y}^{L} \frac{\partial^{2} w 1}{\partial x \partial y}\right. \\
& \left.+N 1_{y}^{L} \frac{\partial^{2} w 1}{\partial y^{2}}+q-k_{o}(w 2-w 1)\right]=0
\end{aligned}
$$

- bottom layer

$$
\begin{aligned}
& -\frac{N 2_{y}^{L}}{R}+\frac{\partial Q 2_{y}^{L}}{\partial y}+\frac{\partial Q 2_{x}^{L}}{\partial x}+\left(1-\mu \nabla^{2}\right)\left[N 2_{x}^{L} \frac{\partial^{2} w 2}{\partial x^{2}}+2 N 2_{x y}^{L} \frac{\partial^{2} w 2}{\partial x \partial y}+N 2_{y}^{L} \frac{\partial^{2} w 2}{\partial y^{2}}\right. \\
& \left.\quad+k_{o}(w 2-w 1)+\left(-k_{w} w 2+k_{p} \nabla^{2} w 2\right)\right]=0
\end{aligned}
$$

\section{Boundary conditions}

All possible boundary conditions have been assumed as simply supported (S), clamped (C) and free $(\mathrm{F})$ edges, which can be written as follows (for the first line $0<x<L_{x}$ and for the second line $\left.0<y<L_{y}\right)$

$$
\begin{array}{lll}
S: & u i=v i=w i=\psi i=M i_{x}=0 & \\
& u i=v i=w i=\varphi &
\end{array}
$$

\section{Solution}

Taking a look on the governing equations, it is clear that a system of nonlinear partial differential equations has been obtained whose solution is not possible to be determined by common analytical methods. In the paper, a new semi-analytical polynomial method (SAPM) is applied, which was presented before by the authors (Dastjerdi et al., 2016c). The SAPM formulation is extremely simple and its accuracy was proved before (Dastjerdi et al., 2016c). According to the mentioned explanations for SAPM, to achieve a solution to the governing equations, the below displacements and rotations can be introduced (Dastjerdi et al., 2016c) ( $i=1,2$ for the upper and bottom layers)

$$
\begin{aligned}
u i= & \sum_{k=1}^{N} \sum_{t=1}^{M} a i_{\{k+t-[1-(k-1)(M-1)]\}} x^{(k-1)} y^{(t-1)} \\
v i= & \sum_{k=1}^{N} \sum_{t=1}^{M} a i_{\{k+t-[1-(k-1)(M-1)]+M N\}} x^{(k-1)} y^{(t-1)} \\
w i= & \sum_{k=1}^{N} \sum_{t=1}^{M} a i_{\{k+t-[1-(k-1)(M-1)]+2 M N\}} x^{(k-1)} y^{(t-1)} \\
\varphi i= & \sum_{k=1}^{N} \sum_{t=1}^{M} a i_{\{k+t-[1-(k-1)(M-1)]+3 M N\}} x^{(k-1)} y^{(t-1)} \\
\psi i= & \sum_{k=1}^{N} \sum_{t=1}^{M} a i_{\{k+t-[1-(k-1)(M-1)]+4 M N\}} x^{(k-1)} y^{(t-1)}
\end{aligned}
$$


By considering Eqs. (5.1) in constitutive equations (3.7)-(3.9), the partial differential equations will be transformed into algebraic equations. The obtained equations can be solved by considering boundary conditions.

\section{Numerical results and discussion}

The curvature radius of the graphene panel has been assumed to tend to infinity in order to obtain a rectangular plate. Then, the results are compared with those by Dastjerdi and Jabbarzadeh (2016). According to Fig. 2, it can be seen that the results are close to each other. The small differences occurred because of tending the radius of the cylindrical panel to infinity. Also, it can be concluded that the differences increase for more flexible boundary conditions. However, the maximum difference is about $2.5 \%$, which is insignificant. Consequently, the obtained results of DQM (Dastjerdi and Jabbarzadeh, 2016) are in compliance with the results of SAPM in this paper.

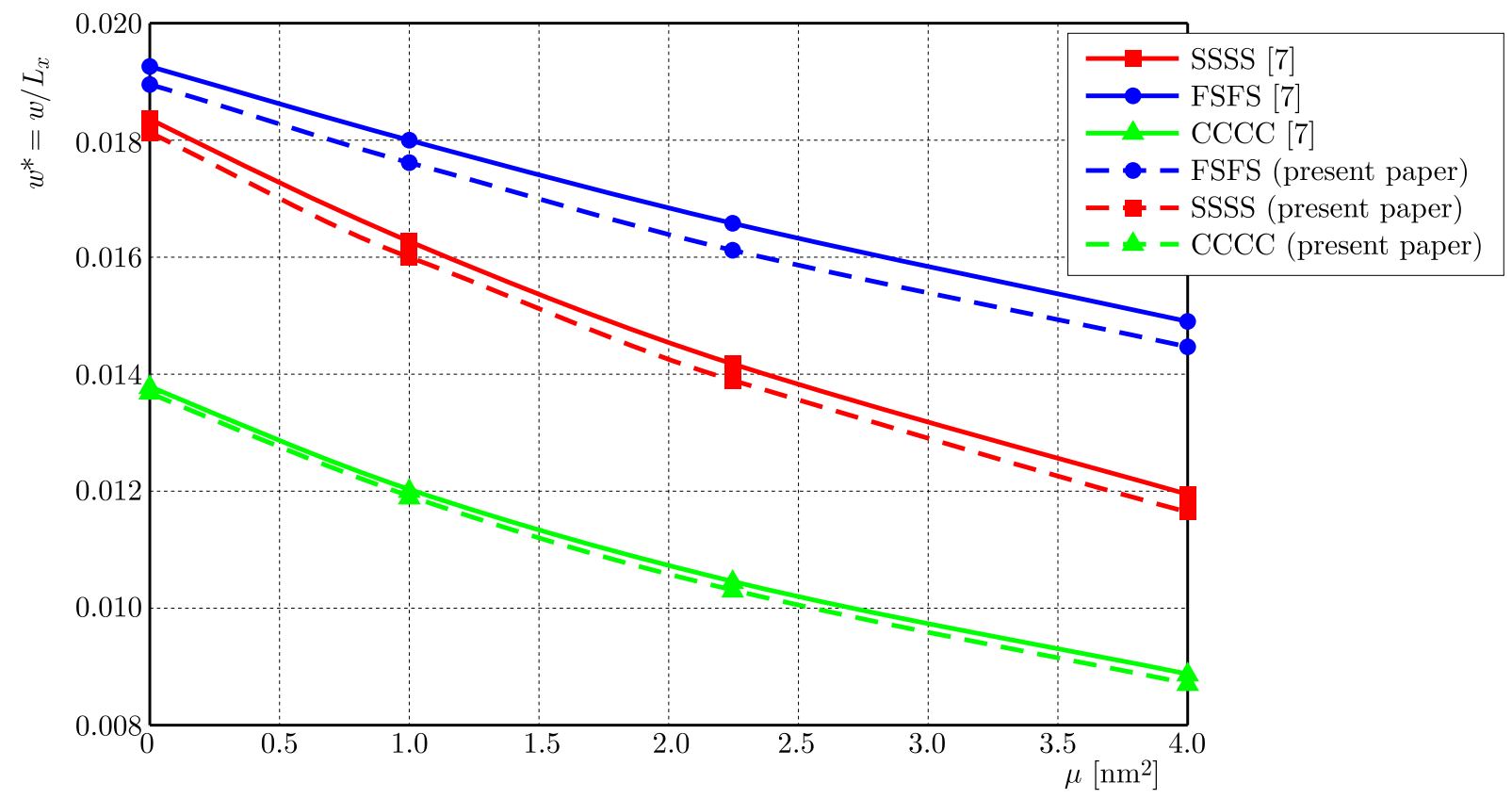

Fig. 2. Comparison between the results of the present paper and Dastjerdi and Jabbarzadeh (2016)

Table 1 indicates the effect of temperature on the results for different types of boundary conditions. It is observed that in higher temperature, the maximum deflection increases. The variations are approximately linear and there is not a considerable difference between CCCC and SSSS boundary conditions. The variations are the same. Also, it is observed that by increasing the nonlocal coefficient, the deflection decreases and the rate of variations remains constant. In CCCC boundary conditions, by increasing the nonlocal effect, the deflection decreases faster, but in SSSS boundary conditions, the variations are small. For example, deflections for $e_{0} a=1 \mathrm{~nm}$ and $e_{0} a=2 \mathrm{~nm}$ are not considerably different, but in CCCC boundary condition, they are different. It may result that in SSSS boundary conditions, due to its more flexibility, temperature raise versus an increase in the small-scale effects is more than in CCCC boundary conditions, and by increasing $e_{0} a$, the deflection has a less descending rate. The properties of the panel are presented below

$$
\begin{array}{lll}
L_{x}=5 \mathrm{~nm} & \theta=\pi & h=0.34 \mathrm{~nm} \quad R=5 \mathrm{~nm} \\
E_{x}=E_{y}=1.06 \mathrm{TPa} & \nu_{x y}=\nu_{y x}=0.3 & q=0.5 \mathrm{GPa} \\
k_{w}=1.13 \mathrm{GPa} / \mathrm{nm} & k_{p}=1.13 \mathrm{Pam} & \alpha=2.02 \cdot 10^{-6} \mathrm{C}^{\mathrm{o}-1}
\end{array}
$$


Table 1. Effect of temperature on nonlocal analysis

\begin{tabular}{|c|c|c|c|c|c|c|}
\hline $\begin{array}{c}\Delta T \\
{\left[\mathrm{C}^{\circ}\right]}\end{array}$ & \multicolumn{5}{|c|}{$w[\mathrm{~nm}]$} & \\
\cline { 2 - 6 } & $e_{0} a=0 \mathrm{~nm}$ & $e_{0} a=1 \mathrm{~nm}$ & $e_{0} a=2 \mathrm{~nm}$ & $R_{m}=\frac{w_{e_{0}^{*} a=1 \mathrm{~nm}}^{*}}{w_{e_{0} a=0 \mathrm{~nm}}}$ & $R_{m}=\frac{w_{e_{0} a=2 \mathrm{~nm}}^{*}}{w_{e_{0}}^{*} a=0 \mathrm{~nm}}$ & \\
\hline \hline 0 & 0.0307 & 0.0294 & 0.0249 & 0.9557 & 0.8086 & \\
100 & 0.0321 & 0.0307 & 0.0262 & 0.9576 & 0.8156 & \multirow{2}{*}{ CCCC } \\
500 & 0.0374 & 0.0362 & 0.0316 & 0.9653 & 0.8441 & \\
1000 & 0.0443 & 0.0432 & 0.0390 & 0.9746 & 0.8800 & \\
\hline 0 & 0.0326 & 0.0318 & 0.0311 & 0.9769 & 0.9560 & \\
100 & 0.0340 & 0.0332 & 0.0325 & 0.9782 & 0.9572 & \multirow{2}{*}{ SSSS } \\
500 & 0.0396 & 0.0389 & 0.0381 & 0.9822 & 0.9616 & \\
1000 & 0.0466 & 0.0461 & 0.0451 & 0.9873 & 0.9657 & \\
\hline
\end{tabular}

To survey the effect of Winkler-Pasternak elastic foundation, Tables 2 and 3 present different types of boundary conditions. In both tables, higher values of elastic the foundation stiffness lead to smaller deflections. As mentioned before, SSSS boundary conditions have more effects on the results, as seen in Table 1. For example, in Table 2, it is considered that by increasing $e_{0} a$ in CCCC boundary conditions, the normal falling rate is available, but in SSSS boundary conditions, the falling rate is smaller. The mentioned variations can be observed in Table 3, too, but it seems that the effect of Pasternak elastic foundation is more evident. Basically, Winkler and Pasternak elastic foundations have a fairly important role in the analysis. It is due to the direct effect of the nonlocal coefficient on the elasticity of Winkler and Pasternak foundations in Eq. (3.9). Consequently, the availability and their values are important in the small scale effect when the nonlocal elasticity theory is applied.

Table 2. Effect of Winkler elastic foundation on the results

\begin{tabular}{|c|c|c|c|c|c|c|}
\hline $\begin{array}{c}k_{w} \\
{[\mathrm{GPa} / \mathrm{nm}]}\end{array}$ & \multicolumn{5}{|c|}{$w[\mathrm{~nm}]$} & \\
\cline { 2 - 6 } & $e_{0} a=0 \mathrm{~nm}$ & $e_{0} a=1 \mathrm{~nm}$ & $e_{0} a=2 \mathrm{~nm}$ & $R_{m}=\frac{w_{e_{0}^{*} a=1 \mathrm{~nm}}^{*}}{w_{e_{0} a=0 \mathrm{~nm}}}$ & $R_{m}=\frac{w_{e_{0}^{*} a=2 \mathrm{~nm}}^{*}}{w_{e_{0} a=0 \mathrm{~nm}}}$ & \\
\hline \hline 0 & 0.0335 & 0.0336 & 0.0330 & 1.0020 & 0.9850 & \\
1 & 0.0316 & 0.0311 & 0.0288 & 0.9841 & 0.9114 & \multirow{2}{*}{ CCCC } \\
5 & 0.0259 & 0.0238 & 0.0188 & 0.9189 & 0.7259 & \\
10 & 0.0210 & 0.0183 & 0.0131 & 0.8714 & 0.6238 & \\
\hline 0 & 0.0354 & 0.0363 & 0.0377 & 1.0254 & 1.0649 & \\
1 & 0.0333 & 0.0335 & 0.0325 & 1.0060 & 0.9759 & SSSS \\
5 & 0.0268 & 0.0255 & 0.0209 & 0.9514 & 0.7798 & \\
10 & 0.0214 & 0.0195 & 0.0144 & 0.9112 & 0.6729 & \\
\hline
\end{tabular}

The van der Waals force is a kind of force which depends on the distance between the layers (Dastjerdi and Jabbarzadeh, 2016). As the distance between two layers decreases, the van der Waals force increases. Figure 3a is drawn for CCCC boundary conditions, and Fig. 3b for SSSS boundary conditions. According to the figures, it is observed that by increasing $k_{0}$, the deflections of two layers approach each other, and after a specified value, an increase in $k_{0}$ has no impact on the results. In both Figs. 3a and 3b, also it is observed that by increasing the nonlocal parameter, the deflections of both layers approach each other faster than before. Therefore, increasing the nonlocal effect results in an increase in van der Waals interaction between the layers. In Fig. 3b, it can be concluded that by increasing small-scale effects, more variation results rather than in CCCC boundary conditions. However, the variations are approximately similar and no considerable change is observed. In this case, the elastic foundation is available and the variations in both cases of CCCC and SSSS boundary conditions are approximately similar. 
Table 3. Effect of Pasternak elastic foundation on the results

\begin{tabular}{|c|c|c|c|c|c|c|}
\hline $\begin{array}{c}k_{p} \\
{[\mathrm{Pam}]}\end{array}$ & \multicolumn{5}{|c|}{$w[\mathrm{~nm}]$} & \\
\cline { 2 - 6 } & $e_{0} a=0 \mathrm{~nm}$ & $e_{0} a=1 \mathrm{~nm}$ & $e_{0} a=2 \mathrm{~nm}$ & $R_{m}=\frac{w_{e_{0}}^{*} a=1 \mathrm{~nm}}{w_{e_{0} a=0 \mathrm{~nm}}}$ & $R_{m}=\frac{w_{e_{0}^{*} a=2 \mathrm{~nm}}^{*}}{w_{e_{0}}^{*} a=0 \mathrm{~nm}}$ & \\
\hline \hline 0 & 0.0335 & 0.0336 & 0.0330 & 1.0029 & 0.9851 & \\
1 & 0.0328 & 0.0321 & 0.0289 & 0.9786 & 0.8811 & \multirow{2}{*}{ CCCC } \\
5 & 0.0302 & 0.0266 & 0.0182 & 0.8808 & 0.6026 & \\
10 & 0.0273 & 0.0216 & 0.0122 & 0.7912 & 0.4469 & \\
\hline 0 & 0.0354 & 0.0363 & 0.0377 & 1.0254 & 1.0650 & \\
1 & 0.0349 & 0.0360 & 0.0367 & 1.0315 & 1.0516 & SSSS \\
2 & 0.0345 & 0.0355 & 0.0347 & 1.0289 & 1.0058 & \\
10 & 0.0302 & 0.0299 & 0.0251 & 0.9900 & 0.8311 & \\
\hline
\end{tabular}
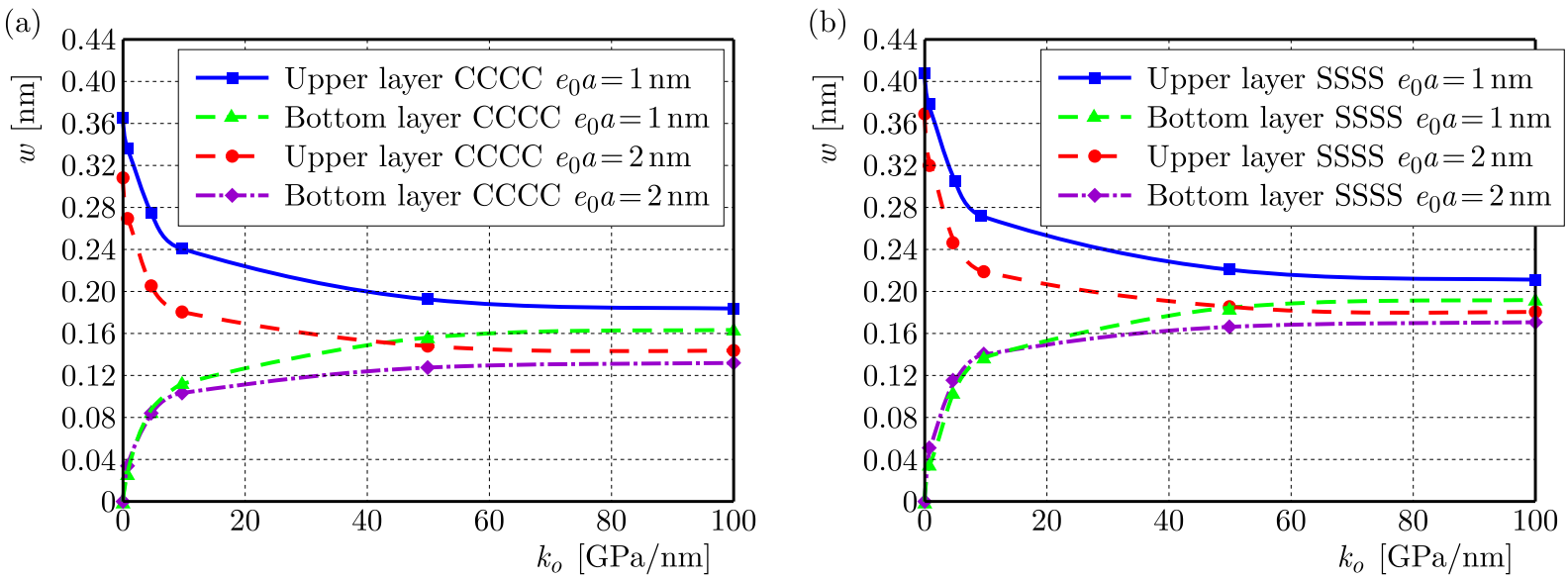

Fig. 3. Variation of deflection versus van der Waals interaction between the layers for (a) CCCC,

(b) SSSS boundary conditions

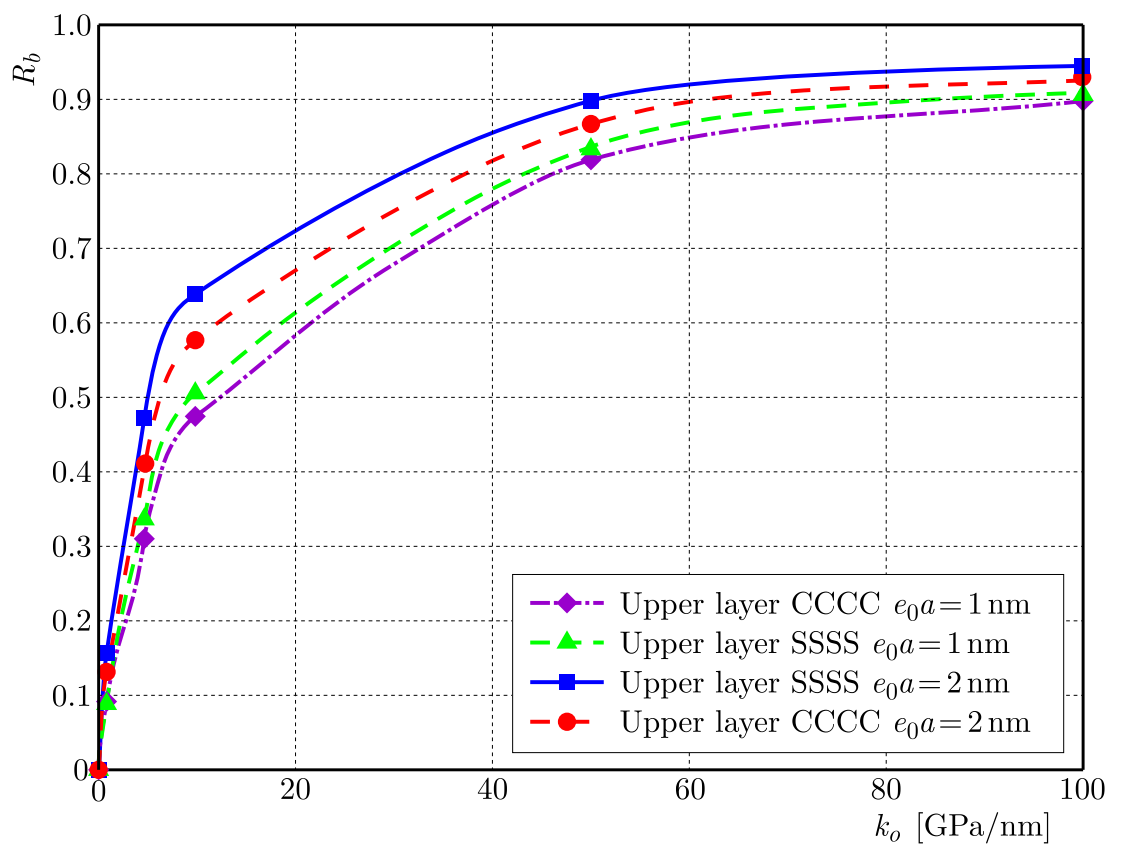

Fig. 4. The effect of van der Waals interaction on the ratio $R_{b}$ 
For better perception, Fig. 4 shows variation of the ratio between the deflections of the bottom layer to the upper layer $R_{b}$ versus an increase in the van der Waals force. It is clearly observed that at the beginning of rising $k_{0}$, the rate of variations is elevated, and in the following, it decreases. Totally, variations in SSSS boundary conditions are greater than in CCCC boundary conditions. This observation results from Figs. 3a and 3b too. Also, by increasing the nonlocal effects, $R_{b}$ becomes greater. In one specified $k_{0}$, which in this case is about $10 \mathrm{GPa} / \mathrm{nm}, R_{b}$ has the maximum distance from other ones in four cases of boundary conditions and nonlocal coefficients.

An increase in radius of the nano graphene shell (R) makes curvature lower and the cylindrical shell transforms into a flat rectangular sheet. Figure 5 shows the effects of increasing $R$ on the results. By increasing $R$, the deflection grows and the rate of variation is high. Continuing, the rate becomes less and remains constant. An infinite value of $R$ means that the cylindrical shell is transformed into a flat rectangular plate. Therefore, by bending the rectangular plate, its deflection subsequently decreases. In SSSS boundary conditions, due to its flexibility, the deflection variations due to $R$ are slow. Also the effect of nonlocal analysis in CCCC boundary conditions is greater than in SSSS boundary conditions. According to Fig. 5, it is observed that when $R$ rises, the value of $R_{m}$ in CCCC boundary conditions decreases more. In this case, the nonlocal effects on the results are more evident (the specifications of panel are similar to Eq. (6.1), only $L_{y}=5 \mathrm{~nm}$ and $\left.\Delta T=0\right)$.

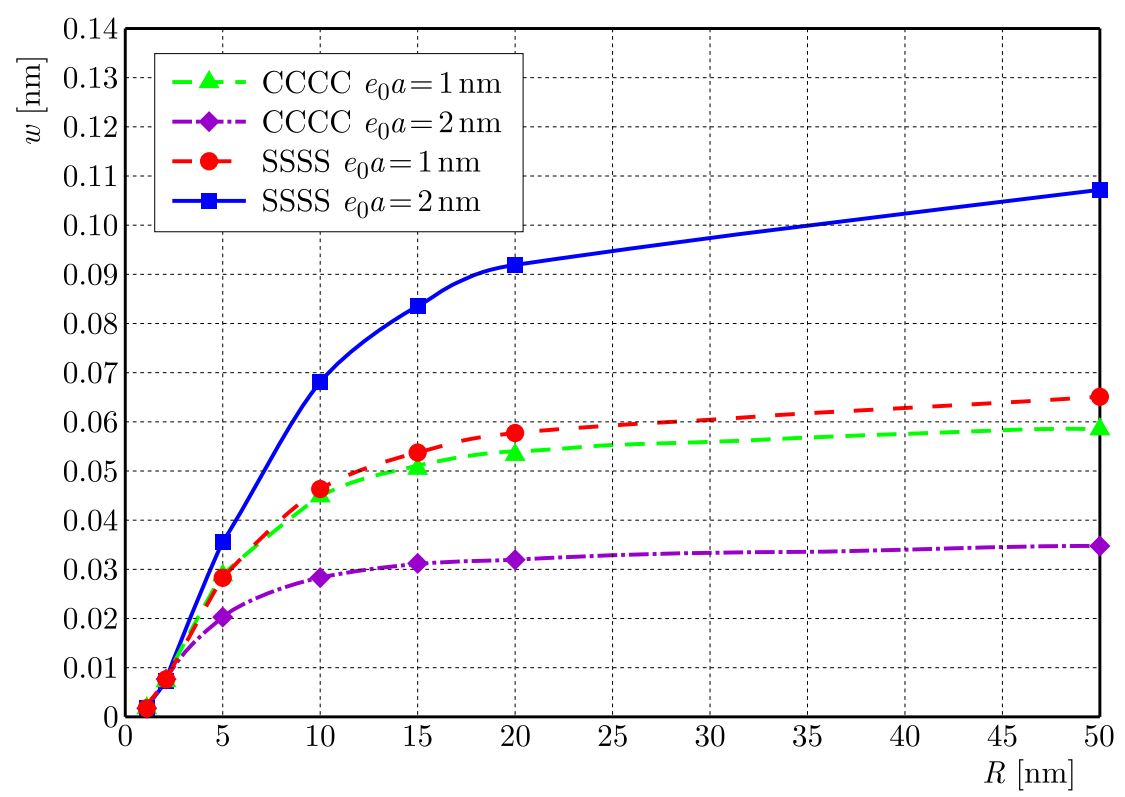

Fig. 5. Deflection due to the increase of radius $R$ of the cylindrical panel

As told before, the nonlocal analysis is more effective in small scales. In this regard, Fig. 6a and $6 \mathrm{~b}$ are drawn. It is observed that by increasing length of the graphene shell, the deflection increases too, and then, the rate of ascending becomes less and remains fixed at one specified value whose further increase in size has no any considerable impact on the results. It is observed that the increasing length results in smaller nonlocal effects, and the effects become greater by decreasing the size. Increasing the size leads to a rise in the ascending rate of reduction of the nonlocal effect, and this conclusion is clearly obvious for higher values of $e_{0} a$. Generally, in small sizes, the nonlocal effects are considerable and nonlocal analysis must be applied. But in larger sizes, the classical theory gives acceptable results by considering a suitable approximation. So, the classical theory is applied for the sizes greater than the nano scale.

Now a nano-tube under internal pressure is studied. This case could be assumed as a shell with radius $R$ whose curvature between both ending edges is $2 \pi$ in $\theta$ direction, so that the 

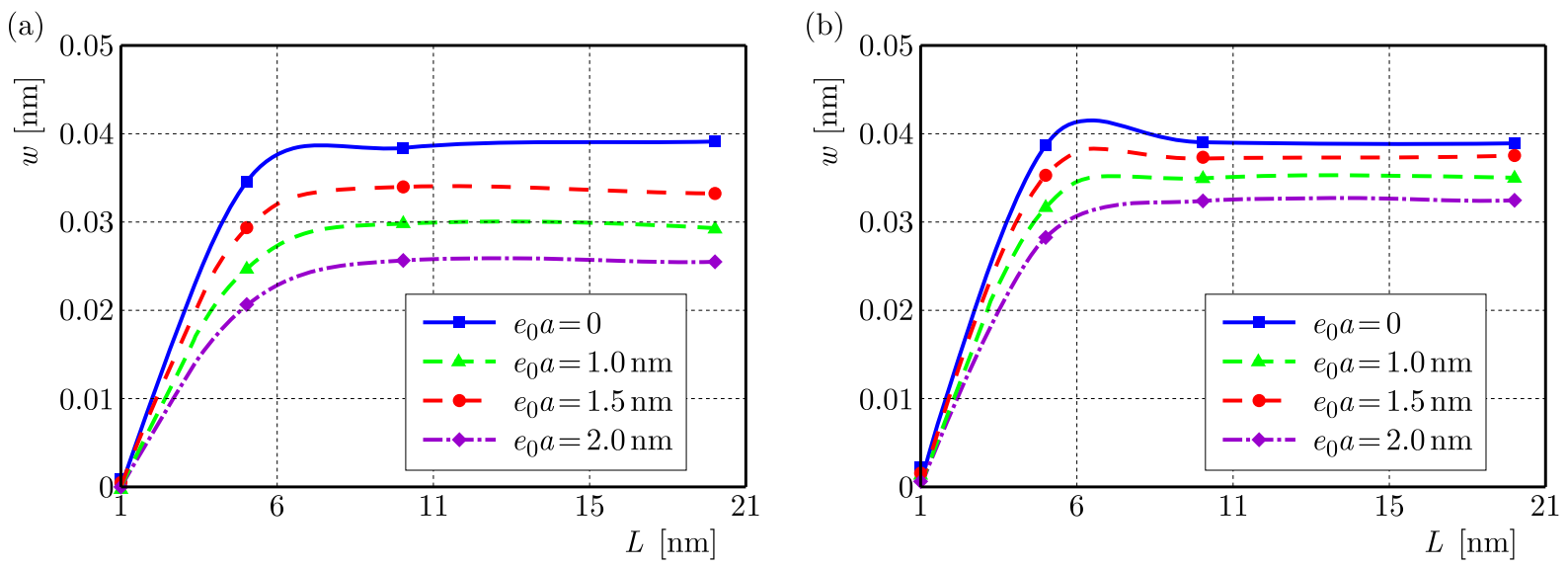

Fig. 6. Variations of deflection versus of the length shell $L_{x}$ for (a) CCCC, (b) SSSS boundary conditions

edges are connected to each other. Nano-tubes can be used widely. For example, to transfer fluid through nano-tubes, the related mechanical properties must be specified. These nano-tubes can be applied in medical purposes such as nano vessels. In this study, the value of expansion and contraction of a nano graphene tube under internal and external pressure are surveyed. At first, a bi-layer nano-tube is studied, whose layers are connected to each other by van der Waals interaction. The ratio of deflection of the bottom layer to the upper layer is defined as $R_{b}$. Now, a bilayer nano-tube is assumed with the specifications given below $\left(R_{b}=w_{\text {bottom layer }} / w_{\text {upper layer }}\right)$

$$
\begin{array}{lll}
L_{x}=15 \mathrm{~nm} & h=0.34 \mathrm{~nm} & E_{x}=E_{y}=1.06 \mathrm{TPa} \\
\nu_{x y}=\nu_{y x}=0.3 & q=0.5 \mathrm{GPa} & k_{w}=1.13 \mathrm{GPa} / \mathrm{nm} \\
p=1.13 \mathrm{Pam} & \Delta T=0 & e_{0} a=1 \mathrm{~nm}
\end{array}
$$

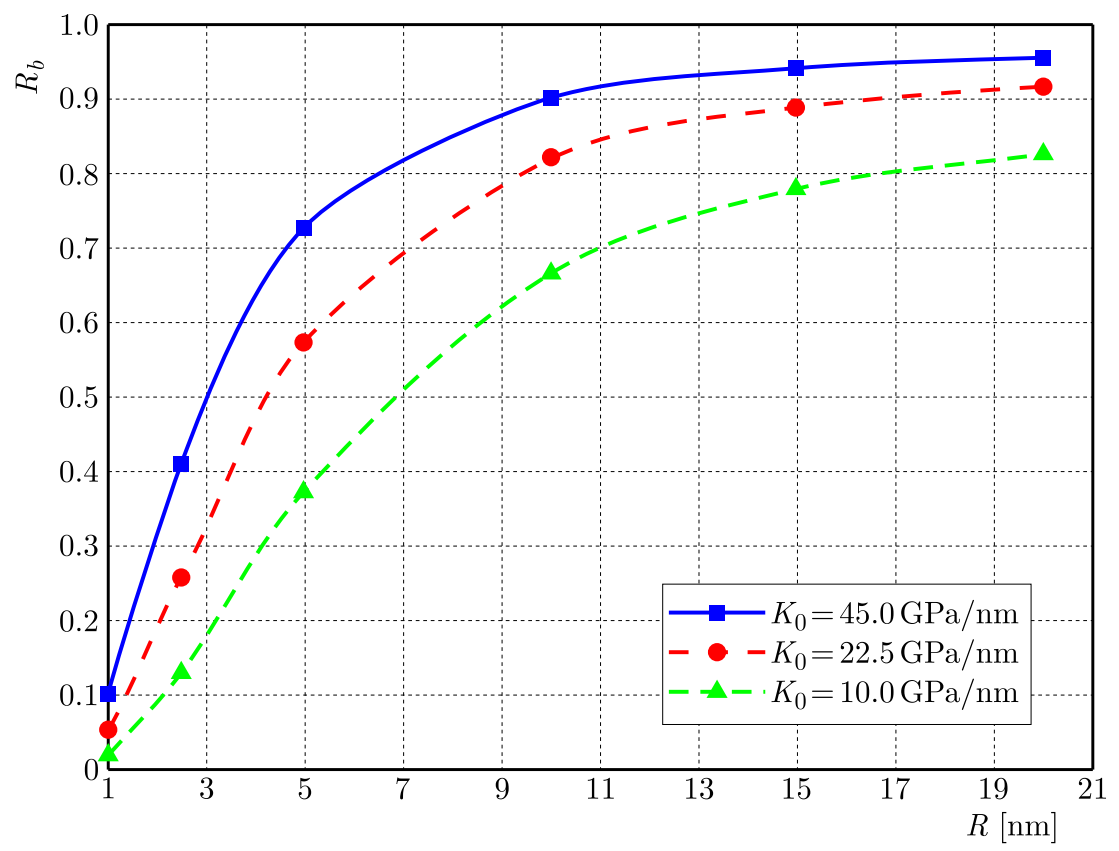

Fig. 7. Variation of nano-tube radius on $R_{b}$ for different van der Waals interactions

Figure 7 shows the effect of increasing radius of the nano-tube on its expansion $R_{b}$ for different values of van der Waals forces. It is observed that by increasing the value of $R$, it leads to an increase in $R_{b}$ too, and in the following, its raising rate lowers and, finally, an increase 
in $R$, does not have any considerable effect on variation of $R_{b}$ any longer. The obtained results are similar with the related results for the shell type studied in Fig. 5. As it is expected, an increase in the van der Waals force has direct effect on the deflection of two bottom and upper layers. By increasing the van der Waals force, these deflections approach each other faster.

To reinforce single layer nano-tubes, bilayer nano-tubes can be applied. To survey the effect of being bilayer nano-tubes on the results, Fig. 8 is presented $\left(R_{b s}=w_{\text {bilayer }} / w_{\text {single layer }}\right)$. Figure 8 shows variation of $R_{b s}$ versus nano-tube radius. As it is observed, by increasing radius $R$, the deflections of both bilayer and single layer nano-tubes approach each other, but for small values of radius $R$, the deflection of the bilayer sheet is considerably less than the single layer one. In this case, strength of the bilayer sheet is greater than the single layer one, but for a larger radius, the strength becomes smaller and the deflection of the bilayer sheet approaches the single layer sheet. Therefore, a single layer nano-tube can be used instead of nano-tube with $R$ radius. Figure 8 is very useful for selection a nano-tube based on the single layer or bilayer one. The figure could be drawn for different conditions, and according to the requirements of the problem, the design must be chosen $\left(k_{o}=45 \mathrm{GPa} / \mathrm{nm}\right)$

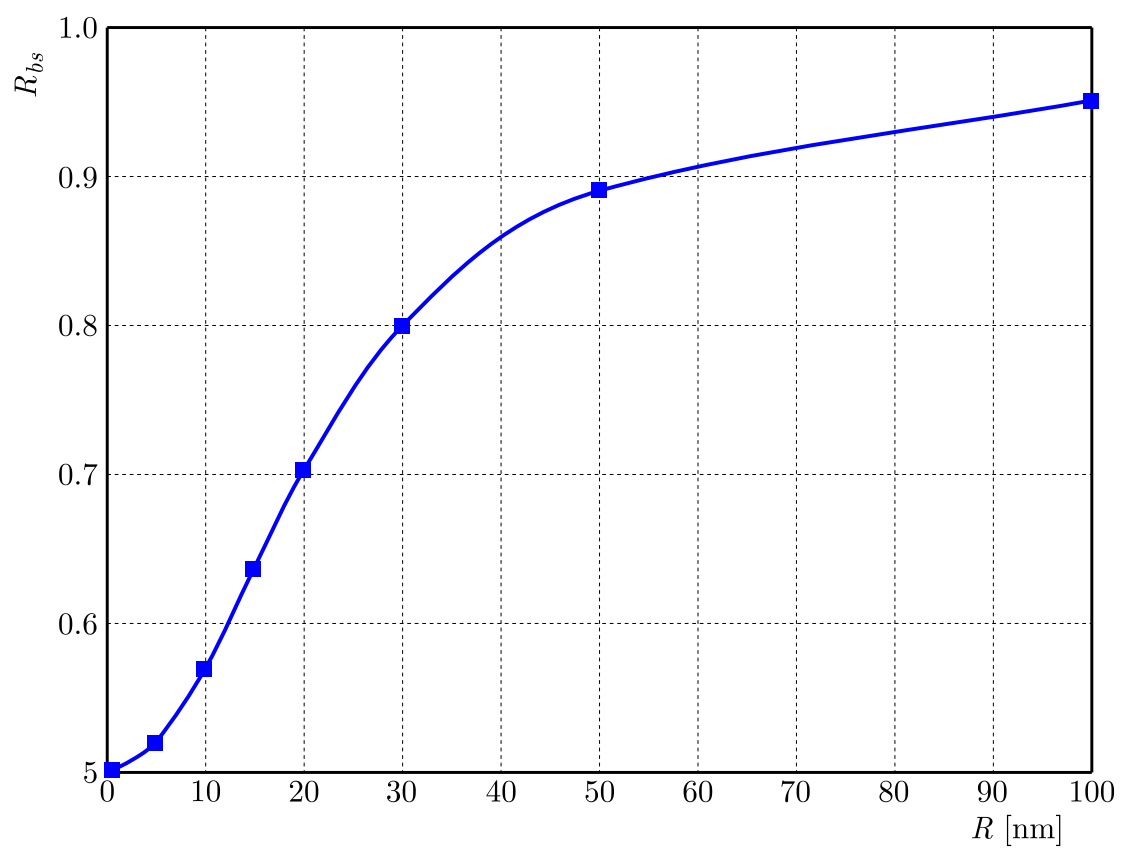

Fig. 8. Single layer to bilayer $R_{b s}$ versus variations of nano-tube radius

\section{Conclusions}

In this paper, for the first time, the mechanical behavior of a graphene cylindrical panel and nano-tube under internal and external pressure is studied. The system is embedded in an elastic matrix and the effect of thermal conduction has been taken into account too. Remarkable conclusions can be listed as follows:

- An increase in the nonlocal effect results in an increase in van der Waals interaction between the layers.

- The small scale effect decreases along the increase of temperature.

- By increasing nonlocal effects, the deflections of the upper and bottom layers approach each other.

- Whatever radius $R$ of the shell is smaller, the nonlocal decrease effects. So, the maximum nonlocal effects occur for rectangular plates (when radius $R$ tends to infinity). 
- An increase in size leads to a rise in the ascending rate of reduction of the nonlocal effect.

- Whatever the radius of nano-tube $R$ rises, the deflection of two layers approach each other.

- The strength of the bilayer nano-tube decreases due to an increase in radius. Consequently, for a high range of radius $R$, a single layer nano-tube can be applied instead of a bilayer one.

\section{Acknowledgement}

I want to dedicate this paper to dr. Jabbarzadeh for his help and my father Reza Dastjerdi who has supported me in every second of my life.

\section{References}

1. Aghababaei R., Reddy J.N., 2009, Nonlocal third-order shear deformation plate theory with application to bending and vibration of plates, Journal of Sound and Vibration, 326, 277-289

2. Ansari R., Faghin Shojaei M., Gholami R., 2016, Size-dependent nonlinear mechanical behavior of third-order shear deformable functionally graded microbeams using the variational differential quadrature method, Composite Structures, 136, 669-683

3. Bisagni Ch., Cordisco P., 2003, An experimental investigation into the buckling and post-buckling of CFRP shells under combined axial and torsion loading, Composite Structures, 60, $391-402$

4. Biswas P., 2014, Thermal stresses, deformations and vibrations of plates and shells - a nonlinear approach, Procedia Engineering, 144, 1023-1030

5. Brush D.O., Almroth B.O., 1975, Buckling of Bars, Plate and Shells, McGraw hill, New York

6. Dastjerdi Sh., Aliabadi Sh., Jabbarzadeh M., 2016a, Decoupling of constitutive equations for multi-layered nano-plates embedded in elastic matrix based on non-local elasticity theory using first and higher order shear deformation theories, Journal of Mechanical Science and Technology, 30, $1253-1264$

7. Dastjerdi Sh., JabBarzadeh M., 2016, Nonlinear bending analysis of bilayer orthotropic graphene sheet resting on Winkler-Pasternak elastic foundation based on non-local continuum mechanics, Composites Part B, 87, 161-175

8. Dastjerdi Sh., Jabbarzadeh M., Aliabadi Sh., 2016b, Nonlinear static analysis of single layer annular/circular graphene sheets embedded in Winkler-Pasternak elastic matrix based on non-local theory of Eringen, Ain Shams Engineering Journal, 7, 873-884

9. Dastjerdi Sh., Lotfi M., Jabbarzadeh M., 2016c, The effect of vacant defect on bending analysis of graphene sheets based on the Mindlin nonlocal elasticity theory, Composites Part B, 98, 78-87

10. Degenhardt R., Kling A., Bethge A., Orf J., Karger L., Rohwer K., Calvi A., 2010, Rolf Zimmermann Investigations on imperfection sensitivity and deduction of improved knock-down factors for unstiffened CFRP cylindrical shells, Composite Structures, 92, 1939-1946

11. Eringen A.C., Edelen D.G.B., 1972, On nonlocal elasticity, International Journal of Engineering Science, 10, 233-248

12. Fazelzadeh S.A., Ghavanloo E., 2014, Vibration analysis of curved graphene ribbons based on an elastic shell model, Mechanics Research Communications, 56, 61-68

13. Hoff N.J., Tsai-Chen S., 1965, Buckling or circular cylindrical shells in axial compression, International Journal of Mechanical Science, 7, 489-520

14. IIJIMA S., 1991, Helical microtubules of graphitic carbon, Nature, 354, 56-58 
15. Khazaeinejad P., Najafizade M.M., Jenabi J., 2010, On the buckling of functionally graded cylindrical shells under combined external pressure and axial compression, Journal of Pressure Vessel Technology ASME, 132-136

16. Lancaster E.R., Calladine C.R., Palmer S.C., 2004, Paradoxical buckling behaviour of a thin cylindrical shell under axial compression, International Journal of Mechanical Science, 42, 843-865

17. Linghai J., Yongliang W., Xinwei W., 2008, Buckling analysis of stiffened circular cylindrical panels using DQ method, Thin Walled Structures, 46, 390-398

18. Martel R., Schmidt T., Shea H.R., Hertel T., Avouris P., 1998, Single and multi-wall carbon nanotube field-effect transistors, Applied Physics Letter, 73, 2447

19. Murmu T., Pradhan S.C., 2009, Vibration analysis of nano-single-layered graphene sheets embedded in elastic medium based on nonlocal elasticity theory, Journal of Applied Physics, 105, 064319

20. Nguyen D., HoAng VAn T., 2010, Nonlinear analysis of stability for functionally graded cylindrical panels under axial compression, Computational Materials Science, 49, S313-S316

21. Postma H.W.C., Teepen T., Yao Z., Grifoni M., Dekker C., 2001, Carbon nanotube singleelectron transistors at room temperature, Science, 293, 76

22. Pradhan S.C., 2009, Buckling of single layer graphene sheet based on nonlocal elasticity and higher order shear deformation theory, Physics Letters A, 373, 4182-4188

23. Reddy J.N., PANG S.D., 2008, Nonlocal continuum theories of beams for the analysis of carbon nanotubes, Journal of Applied Physics, 103, 023511

24. Tans S.J., Devoret M.H., Dai H., Thess A., Smalley R.E., Geerligs L.J., Dekker C., 1997, Individual single-wall carbon nanotubes as quantum wires, Nature, 386, 474-477

25. WANG Q., WANG C.M., 2007, The constitutive relation and small scale parameter of nonlocal continuum mechanics for modeling carbon nanotube, Nanotechnology, 18, 1-10

26. Zhang L.W., Zhang Y., Zou G.L., Liew K.M., 2016, Free vibration analysis of triangular CNT-reinforced composite plates subjected to in-plane stresses using FSDT element-free method, Composite structures, 149, 247-260

27. Zhang Y., Lei Z.X., Zhang L.W., Liew K.M., Yu J.L., 2015, Nonlocal continuum model for vibration of single-layered graphene sheets based on the element-free kp-Ritz method, Engineering Analysis with Boundary Elements, 56, 90-97

28. Zhaо X., LiEw K.M., 2009, Geometrically nonlinear analysis of functionally graded shells, International Journal of Mechanical Science, 51, 131-144 